\title{
Near-resonance enhanced label-free stimulated Raman scattering microscopy with spatial resolution near $130 \mathrm{~nm}$
}

\author{
Yali Bi ${ }^{1,2,3}$, Chi Yang ${ }^{1,2}$, Yage Chen ${ }^{1,2}$, Shuai Yan ${ }^{1,2}$, Guang Yang ${ }^{1,2}$, Yaozu Wu ${ }^{1}$, Guoping Zhang ${ }^{3}$ and Ping Wang ${ }^{1,2}$
}

\begin{abstract}
High-resolution optical microscopes that can break $180 \mathrm{~nm}$ in spatial resolution set to conventional microscopies are much-needed tools. However, current optical microscopes have to rely on exogenous fluorescent labels to achieve high resolution in biological imaging. Herein, we report near-resonance enhanced label-free stimulated Raman scattering (SRS) microscopy with a lateral resolution near $130 \mathrm{~nm}$, in which the high-resolution image contrast originates directly from a low concentration of endogenous biomolecules, with sensitivity gains of approximately 23 times. Moreover, by using a 0.3-m-long optical fiber, we developed hyperspectral SRS microscopy based on spectral focusing technology. Attributed to enhancements in spatial resolution and sensitivity, we demonstrated highresolution imaging of three-dimensional structures in single cells and high-resolution mapping of large-scale intact mouse brain tissues in situ. By using enhanced high-resolution hyperspectral SRS, we chemically observed sphingomyelin distributed in the myelin sheath that insulates single axons. Our concept opens the door to biomedical imaging with $\sim 130 \mathrm{~nm}$ resolution.
\end{abstract}

\section{Introduction}

Label-free and high-resolution optical microscopes that can directly identify and image native biomolecules are highly desired ${ }^{1-4}$ but remain challenging. Advanced nonlinear imaging modalities, including pump-probe, four-wave mixing ${ }^{5-7}$, coherent anti-Stokes Raman scattering $(\mathrm{CARS})^{8-13}$ and stimulated Raman scattering $(\mathrm{SRS})^{14,15}$ microscopies, have been proposed in different approaches to improve spatial resolution, but only a few have been found to be very effective for biological systems.

Correspondence: Ping Wang (p_wang@hust.edu.cn) or

Guoping Zhang (gpzhang@mail.ccnu.edu.cn)

${ }^{1}$ Britton Chance Center for Biomedical Photonics, Wuhan National Laboratory for Optoelectronics-Huazhong University of Science and Technology, Wuhan, Hubei 430074, China

${ }^{2}$ MoE Key Laboratory for Biomedical Photonics, Collaborative Innovation Center for Biomedical Engineering, School of Engineering Sciences, Huazhong University of Science and Technology, Wuhan, Hubei 430074, China Full list of author information is available at the end of the article.

These authors contributed equally: Yali Bi, Chi Yang
For fluorescence imaging, it is straightforward to use nonlinear multiphoton microscopy for attaining an increase in imaging resolution of $\sqrt{2}$ or more because the fluorescent signal is generated only at the very center of the focal spot of the laser ${ }^{16}$. However, the excitation laser wavelengths are strictly limited in the near-infrared (NIR) region because ultraviolet (UV) dyes or fluorescent proteins applicable for visible and nonlinear excitation are not readily available. In addition, visible femtosecond laser systems are not commercially available. Thus, the potential improvement in spatial resolution is completely compromised by the long wavelength adopted for nonlinear fluorescence imaging.

Fortunately, nonlinear CARS and SRS microscopies are free of limitations from labeling and applicable to this spot reduction effect. To fully utilize the nonlinear advantage to defeat the resolution limit, we reduced the wavelengths of our femtosecond lasers to the visible region ${ }^{17}$ and demonstrated visible SRS microscopy with subdiffraction resolution down to $130 \mathrm{~nm}$. Meanwhile, the sensitivity of 
SRS increased by 23 times owing to near resonance and increased photon energy. Moreover, we adopted a 0.3-mlong polarization-maintaining single-mode (PM-SM) optical fiber to ensure excellent beam quality for highresolution imaging and, importantly, achieved spectral focusing based hyperspectral SRS for selectively imaging biomolecules in intact tissues.

\section{Results}

In the proof of concept of our high-resolution SRS microscope, the laser module outputs two femtosecond laser lines at wavelengths of 900 and $1040 \mathrm{~nm}$ (Fig. 1a, see setup details in the Materials and methods section). We effectively doubled the laser frequencies of our NIR femtosecond lasers by two beta-barium borate (BBO) crystals, with their wavelengths reduced in half to 450 and $520 \mathrm{~nm}$, which served as pump and Stokes lasers, respectively. Figure $1 \mathrm{~b}$ illustrates the energy diagram of our proposed concept. Since the nonlinear SRS signal is generated at the very center of the focal spot and complies with quadratic dependence of the excitation intensities, the spatial resolution naturally gains an additional $\sqrt{2}$ in visible SRS imaging. Thus, the spatial resolution of this system determined by the Rayleigh criterion can be

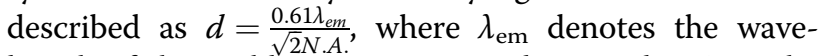
length of the visible SRS excitation laser and N.A. is the numerical aperture of the applied objective. As we used a high-power oil immersion objective (N.A. 1.49) and short wavelength $\left(\lambda_{\mathrm{em}}=450 \mathrm{~nm}\right)$ for excitation, the theoretical spatial resolution of our system can reach $130 \mathrm{~nm}$. As shown in Fig. 1c, we performed visible SRS imaging for 60 $\mathrm{nm}$ polystyrene (PS) beads by detecting carbon-hydrogen $(\mathrm{CH})$ vibrations at $\sim 3050 \mathrm{~cm}^{-1}$ (Supplementary Fig. 3a); the cross-section profile of one bead is presented in Fig. 1d. The size of the full width at half maximum (FWHM) is $\sim 114 \mathrm{~nm}$ by Gaussian fitting (see more experimental results in Supplementary Fig. 1e, f). After deconvolution of the actual size of the PS beads, we determined the point spread function (PSF) of our system to be $\sim 107 \mathrm{~nm}$. The spatial resolution defined by the Rayleigh criterion, which states the distance between two resolvable spots, is $\sim 1.2$ times the FWHM of the PSF $^{18-20}$. In contrast to the high-resolution images obtained by the visible SRS microscope, the average FWHM of the PS beads imaged by NIR SRS was approximately $300 \mathrm{~nm}$ with a $60 \times$ water immersion objective (N.A. 1.2), and improved to $240 \mathrm{~nm}$ with a $100 \times$ oil immersion objective (N.A. 1.49, Supplementary Fig. 1a-d).

In addition to the improved spatial resolution, the increased photon energy also enhanced the SRS imaging sensitivity to endogenous biomolecules. In a conventional SRS microscope implementing NIR lasers to drive a coherent Raman transition, the intermediate virtual state is far detuned from the electronic excited state of the biomolecules. Here, we employed a visible laser to drive the near resonant stimulated Raman transition which, in theory, improves the coherent transition efficiency by approximately 50 times (see detailed calculation in the Materials and methods section). As a result, the laser powers required for further SRS imaging of biological samples are less than $5 \mathrm{~mW}$ for both the pump and Stokes beams.

To achieve molecular specificity along with improved imaging resolution and sensitivity, we implemented spectral focusing based hyperspectral SRS imaging in our concept $^{21,22}$. Since the optical fiber shows a particularly high dispersion in the visible wavelength range, we adopted a 0.3-m-long PM-SM fiber to chirp the femtosecond laser pulses instead of applying high-dispersion glass rods. Importantly, the PM-SM fiber exhibits multiple advantages which ensures absolute collinearity for the pump and Stokes lasers, stabilized beam pointing and polarization, and an excellent Gaussian beam profile for high-resolution hyperspectral SRS imaging. To test and calibrate the proposed spectral focusing system, we measured the SRS spectra for dimethyl sulfoxide (DMSO) and methanol $(\mathrm{MeOH})$ within a spectral window between 2800 and $3050 \mathrm{~cm}^{-1}$ (Fig. 1e). Considering that the laser energy has a certain distribution with wavelength, it is necessary to calibrate the SRS intensity in different Raman shifts $^{23}$. After spectrum normalization by the transient absorption signal measured from folic acid (FA), the Raman bands of DMSO (2918 and $3000 \mathrm{~cm}^{-1}$ ) and $\mathrm{MeOH}$ (2835 and $2947 \mathrm{~cm}^{-1}$ ) were found to coincide with the spontaneous Raman spectra shown in Supplementary Fig. 3b.

After addressing challenges in improving spatial resolution, sensitivity, and molecular specificity, we demonstrated high-resolution SRS imaging of single cells. Figure 2a shows a vibrational image of cultured neurons acquired by a traditional NIR SRS microscope. As a direct comparison, the neurons imaged by the visible SRS microscope exhibit a significantly improved image contrast and spatial resolution, as shown in Fig. 2b. We observed the fine structures of the neurons which stretched out multiple dendrites with a clear boundary. Meanwhile, intracellular lipid droplets with different sizes were widely spotted in the soma and dendrites. It is worth noting that we also observed large lipid droplets in cultured neurons (pinpointed by a yellow arrow, inset in Fig. 2b) which were rarely found in the neurons in brain tissues. In Fig. 2c, we visualized the ultrafine spines distributed along the dendrites, as indicated by green arrows (microtubule-associated protein 2 (MAP2) immunolabeled, see fluorescence and visible SRS images in Supplementary Fig. 4a, b). Interestingly, two glia cells were also observed by label-free SRS imaging, with each of them remaining close to one neuron. In addition, it is 
a

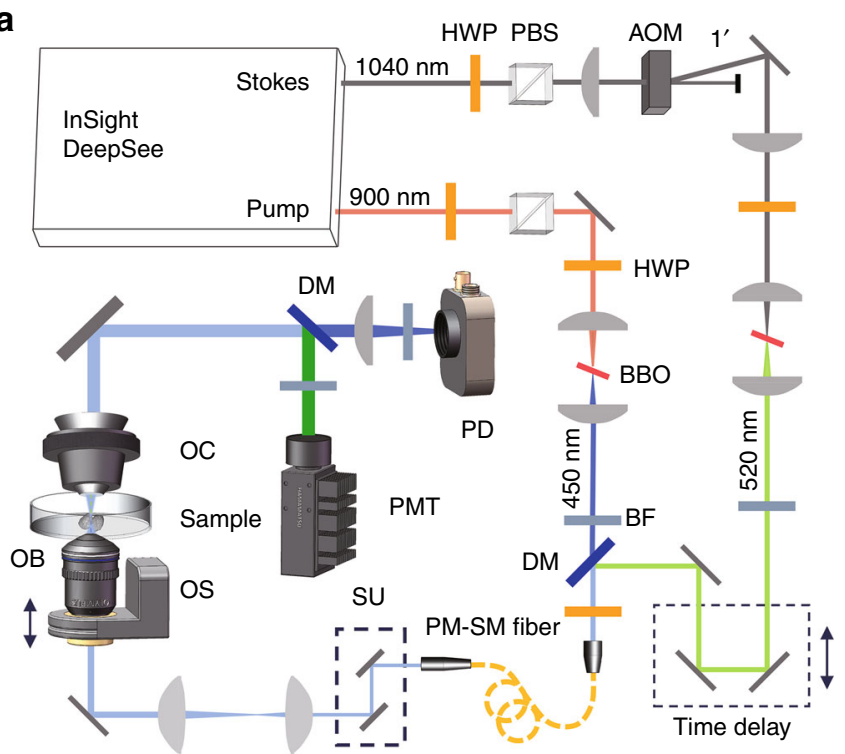

C 60

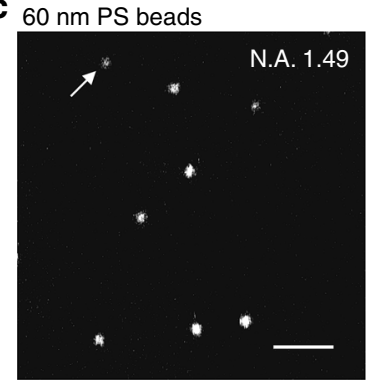

d

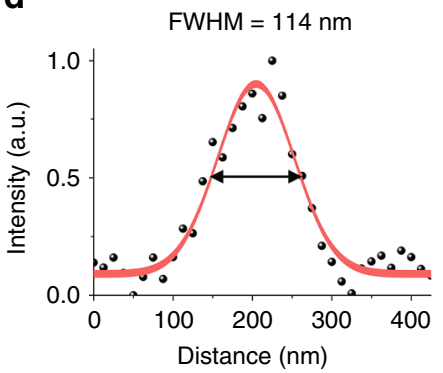

b

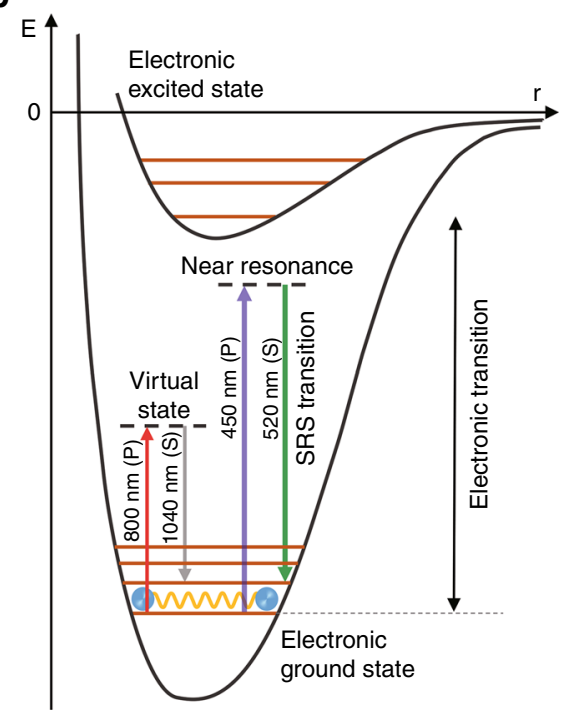

e

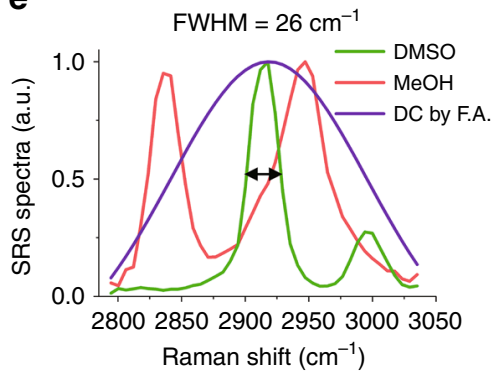

Fig. 1 Visible SRS microscopy and spectroscopy. a Schematic of the visible SRS imaging system. HWP half wave plate, AOM acousto-optical modulator, BF bandpass filter, DM dichroic mirror, SU scanning unit, OS objective scanner, OB objective, OC oil condenser, PD photodiode, PMT photomultiplier tube, PM-SM polarization-maintaining single-mode fiber. $\mathbf{b}$ Energy diagram. $\mathbf{c}$ Visible SRS image of PS beads. $\mathbf{d}$ Cross-section profile of a selected PS bead in the boxed region in (c). The Gaussian fitting measured size of the bead is $114 \mathrm{~nm}$ (FWHM). e SRS spectra for MeOH and DMSO, which were normalized by the pump-probe signal of folic acid (FA) after Gaussian fitting. Scale bar, $1 \mu \mathrm{m}$

known that neurons are very active toward sensing and contacting with their neighbors. Strikingly, even for young neurons that were cultured in Petri dishes for only 7 days, densely distributed neural networks and connections could be clearly visualized (Supplementary Fig. 4c, d).

After installation of an oil immersion high N.A. (1.49, $100 \times$ ) objective in the visible SRS system, we performed visible SRS imaging of U2OS cells (Fig. 2d, e). In comparison, the visible SRS image exhibited a substantially higher spatial resolution; we could visualize very fine mesh structures located outside of the cell nucleus which probably were endoplasmic reticulum (see more images in Supplementary Fig. 5). In Fig. 2f, we plotted the intensity profile across a lipid droplet, with the Gaussian fitted FWHM found to be $113 \mathrm{~nm}$ (Fig. 2g). By direct comparison, we conclude that the spatial resolution of the visible SRS microscope is significantly improved compared to traditional SRS.
A nonlinear SRS microscope naturally possesses the capability of optical sectioning; thus, we performed highresolution three-dimensional (3D) SRS imaging of HeLa cells cultured in a Petri dish. In Fig. 3a, the cell body mostly located close to the planar glass surface $(Z=0 \mu \mathrm{m})$ was depicted in great detail. Actually, the cell body did not simply appear as a flat structure on the surface but rather was presented as a 3D structure. In the region indicated by the white arrow, we observed many irregular protrusions toward the surface of the glass which were probably responsible for cell migration as "feet". In 3D stacked images (Fig. 3c), these tiny protrusions were extended out to approximately $1 \mu \mathrm{m}$ in height from their cell body (see image at $Z=1.5 \mu \mathrm{m}$ ). In addition to these vertical protrusions, we also clearly visualized the delicate filopodia extending out horizontally from the basal membrane located on both sides of the cell body (indicated by green arrows) which may play essential roles in the sensing of 

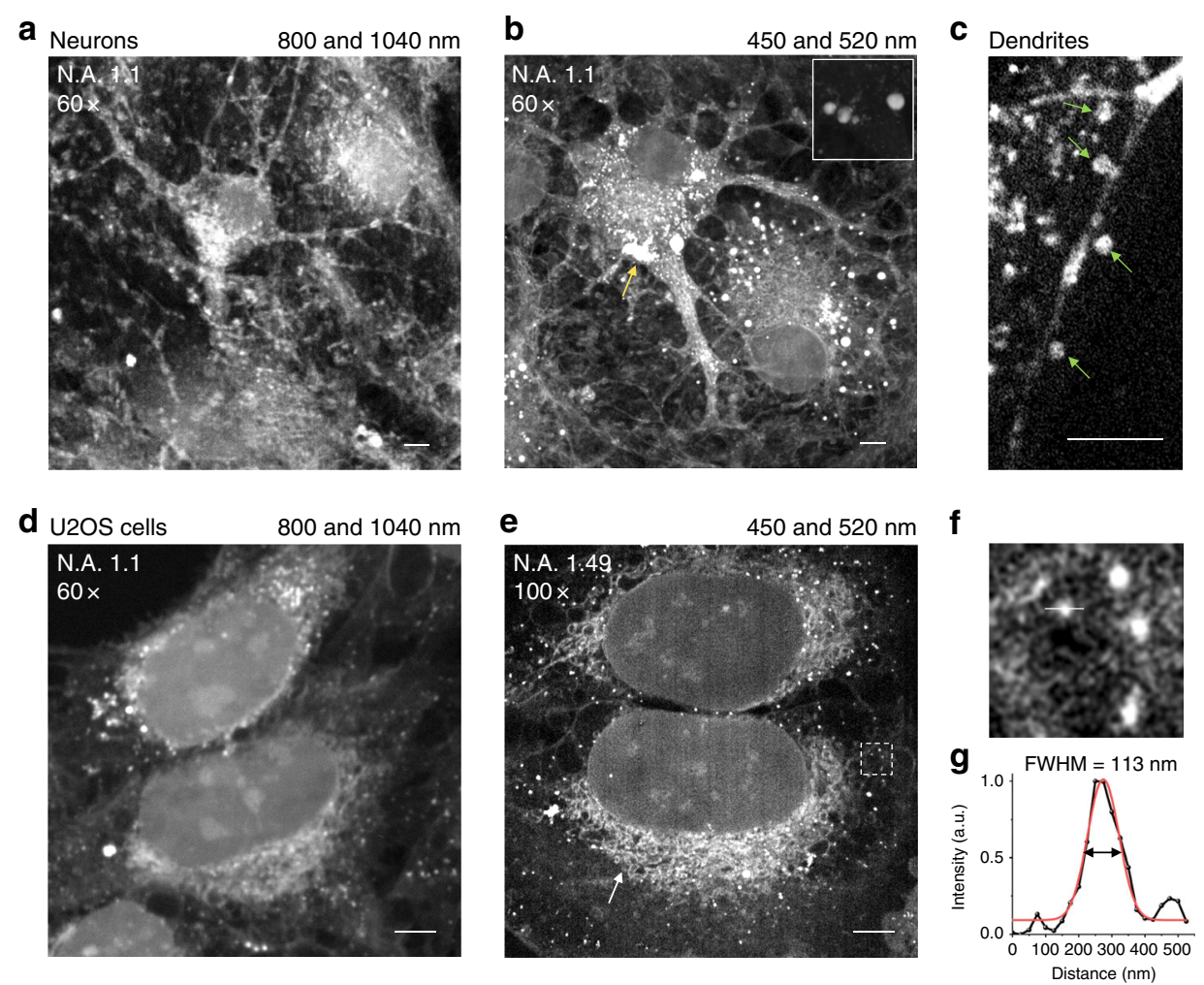

Fig. 2 Visible SRS imaging of cultured neurons and U2OS cells. a, b Direct comparison of neuron images obtained by a conventional NIR SRS microscope (a) and visible SRS microscope (b). c Dendritic spines of the neuron. $\mathbf{d}$, e NIR SRS image (d) and visible SRS image (e) of U2OS cells. $\mathbf{f}$ Zoom-in image of boxed area in (e). $\mathbf{g}$ Intensity profile of the indicated lipid droplet in U2OS cell in (f). The red curve shows the Gaussian fitting result for a lipid droplet size with a FWHM of $113 \mathrm{~nm}$. Scale bars, $5 \mu \mathrm{m}$

surrounding environments. These cell filopodia are approximately $0.2 \mu \mathrm{m}$ in diameter and $10 \mu \mathrm{m}$ or less in length. Figure $3 \mathrm{~b}$ shows a high-resolution SRS image of the nuclei of the same cell at $Z=12.5 \mu \mathrm{m}$. From the imaging planes scanned at higher positions between $Z=8$ and $21 \mu \mathrm{m}$, we clearly observed 3D structures for both nuclear envelopes and the nucleolus with great detail (Fig. 3d). The complete 3D structures of the cells are presented in Supplementary Fig. 6 and Supplementary Video.

In fact, in contrast to NIR lasers, the visible lasers will cause severe photodamage to cells. In Supplementary Fig. 2, we characterized the level of photodamage to live HeLa cells as a function of total laser power and continuous laser scanning time. We found that the laser scanning time for safe live cell imaging decayed exponentially with increasing total laser power. When the total laser power was lower than $2.5 \mathrm{~mW}$, the living cells could tolerate continuous laser scanning without a time limit. Thus, we have to balance the laser power and photodamage for extended live cell imaging. However, for fixed cells, we did not observe any apparent photodamage or thermal damage when the total laser power was below $10 \mathrm{~mW}$.
Due to difficulties in elimination of the out-of-focus fluorescence background, high-resolution imaging of intact tissue is challenging for fluorescence microscopes. To demonstrate the capability of direct tissue imaging, we demonstrated high-resolution SRS imaging of unprocessed brain tissue from a C57 mouse. As shown in Fig. 4a, a long strip area covering the cortex, alveus of the hippocampus (alv), subiculum $(S)^{24}$, and other brain areas was examined (see detailed atlas of the mouse brain in Supplementary Fig. 7). In Fig. 4b, c, we present highresolution SRS images of a neuron and part of one blood vessel in brain tissue (their relative locations are indicated in Fig. 4a). In the white matter of the brain, we observed a high density of myelinated axons (Fig. 4d). Since the myelin sheaths surrounding the axons are dominated by lipids, the SRS signal is much stronger than that from the other parts. Thus, in visible SRS imaging, the axons appeared as enclosed circles with a diameter of approximately $1 \mu \mathrm{m}$ on the cross-section and two parallel curves on the longitudinal section. As shown in Fig. 4e, the neural networks were weaved of crisscrossed fiber bundles (indicated by yellow arrows), among which blood vessels and neurons were vastly distributed. Especially in the alv 


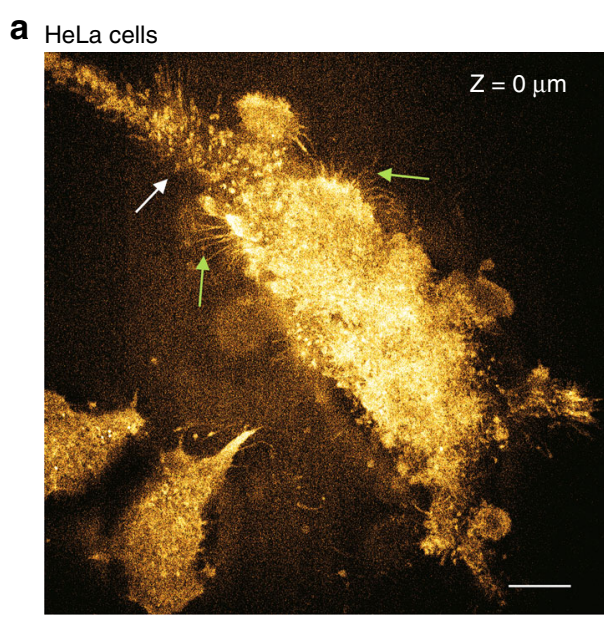

C $\mathrm{Z}=0 \mu \mathrm{m}$

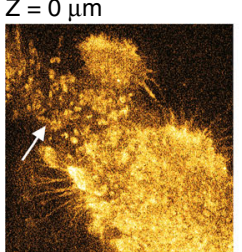

d $Z=7.5 \mu \mathrm{m}$

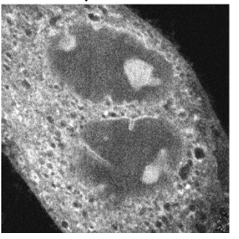

$0.5 \mu \mathrm{m}$

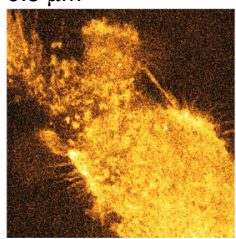

$9.0 \mu \mathrm{m}$

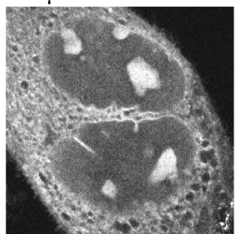

b

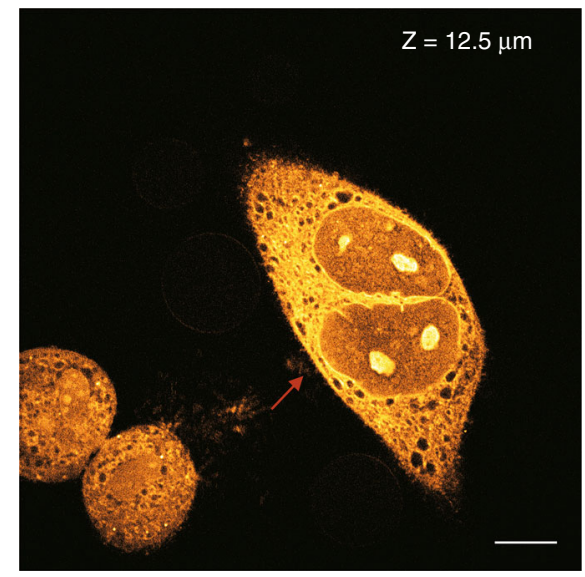

$1.0 \mu \mathrm{m}$

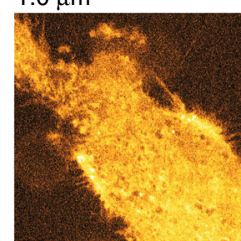

$1.5 \mu \mathrm{m}$

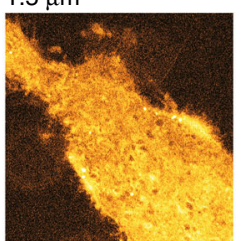

$2.0 \mu \mathrm{m}$

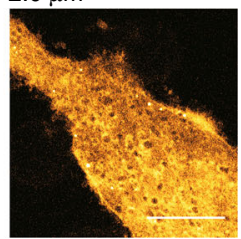

$12.5 \mu \mathrm{m}$

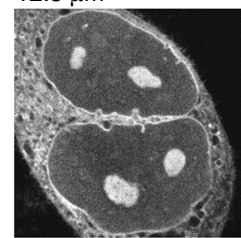

$16.0 \mu \mathrm{m}$

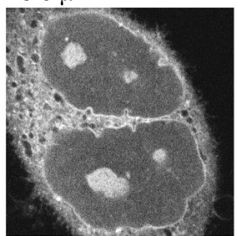

$21.0 \mu \mathrm{m}$

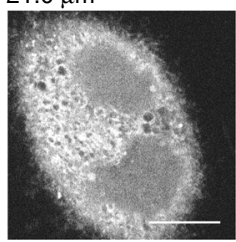

Fig. 3 3D visible SRS imaging of cultured HeLa cells. $\mathbf{a}$, $\mathbf{b}$ SRS images of cell body located mostly close to the planar glass surface (a) and at a height of $Z=12.5 \mu \mathrm{m}(\mathbf{b})$. Water immersion objective with N.A. 1.1 was used for imaging. $\mathbf{c}$ Zoom-in images of the cell body at different heights corresponding to the location indicated by a white arrow in (a). $\mathbf{d}$ Images of cell nuclei at different heights corresponding to the location indicated by the red arrow in (b). Scale bars, $10 \mu \mathrm{m}$

region, fiber bundles of axons were packed with unprecedented density in all directions (Fig. 4f). We also observed a clear boundary that divided alv and $S$ regions, where the distribution density of the somas and axons exhibited great differences. In the $S$ region, we found densely populated neurons (indicated by red arrows), but much fewer fiber bundles. The high-resolution SRS maps covering the complete inspected area of the brain tissue (indicated in Fig. 4a) are exhibited in Supplementary Fig. 9. To evaluate the imaging depth for visible SRS imaging, we performed 3D imaging of white matter in a tissue slice of mouse brain. As shown in Supplementary Fig. 8, we directly compared the imaging depth of our system with that of an NIR SRS system. We found that the visible SRS imaging depth was approximately $10 \mu \mathrm{m}$ with decent image contrast. For the NIR SRS microscope, the penetration depth is approximately $50 \mu \mathrm{m}$ in a similar region.
Based on significantly improved spatial resolution, we further performed hyperspectral SRS imaging of mouse brain tissue ${ }^{25}$. It is known that the major chemical compositions of brain tissues are sphingomyelin, neutral lipids, and protein. As shown in Fig. 5a, sphingomyelin comprises two straight long acyl chains in its chemical structure. To differentiate the major chemical compounds in mouse brain tissue, we acquired SRS spectra for pure chemicals including sphingomyelin, glyceryl trioleate (TO), and bovine serum albumin (BSA, a protein representative), as shown in Fig. 5b. Except for the symmetric $\mathrm{CH}_{2}$ vibrational band at $2853 \mathrm{~cm}^{-126}$, TO exhibits a distinctive Raman feature for lipid unsaturation at $3005 \mathrm{~cm}^{-1}$ which arises from vibrational stretching of $=\mathrm{CH}^{27-29}$. In contrast to unsaturated $\mathrm{TO}$, sphingomyelin presents a characteristic Raman band at $2883 \mathrm{~cm}^{-1}$ which is the signature band for saturation that possibly originated from Fermi resonance or asymmetric vibration of $\left(\mathrm{CH}_{2}\right)_{\mathrm{n}}$ 

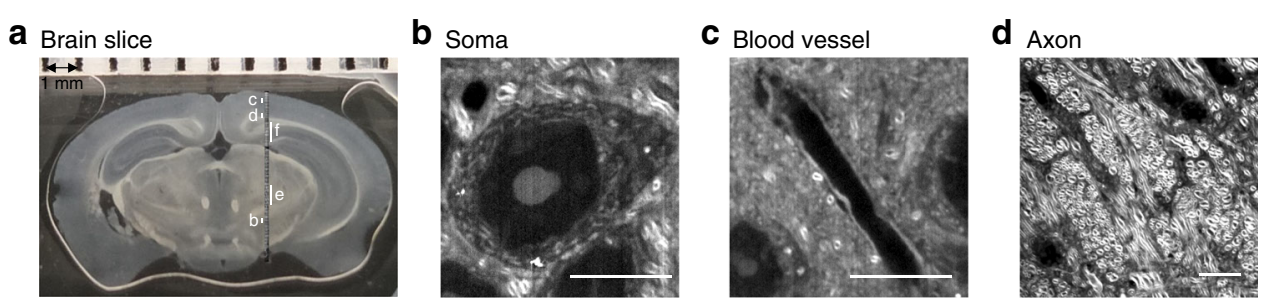

e

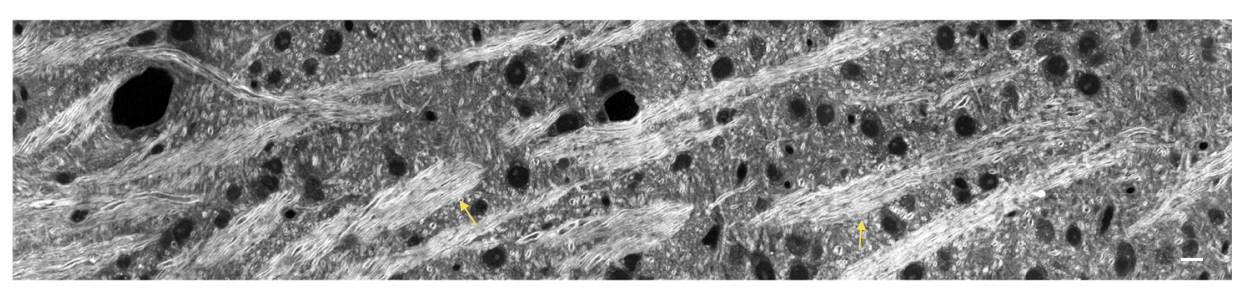

f Alveus of the hippocampus Subiculum

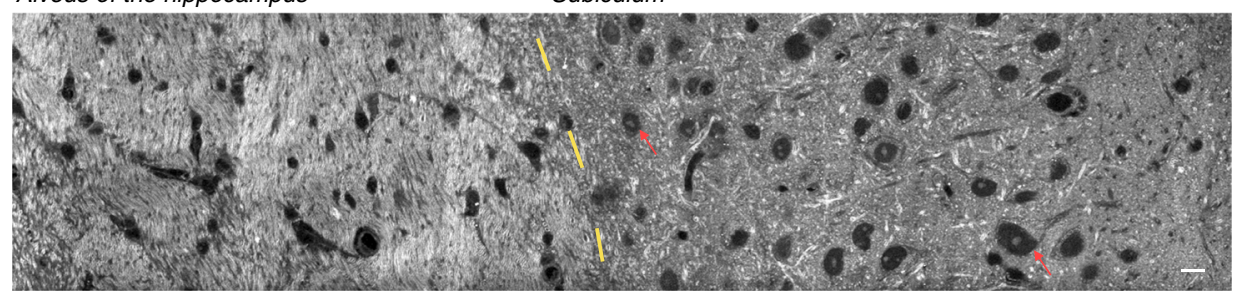

Fig. 4 Visible SRS imaging of an unprocessed brain tissue section from a C57 wild-type mouse. a Overview of a coronal section of the brain slice. SRS inspected area is shown. b-d Enlarged views that illustrate the architectures of the soma (b), blood vessel in the cortex (c), and fiber bundles in white matter $(\mathbf{d})$, with their locations marked by white lines $(\mathbf{b}-\mathbf{d})$ in $(\mathbf{a})$, respectively. $\mathbf{e}, \mathbf{f}$ High-resolution SRS imaging of brain areas corresponding to $(\mathbf{e}, \mathbf{f})$ indicated in $(\mathbf{a})$, respectively. Scale bars, $10 \mu \mathrm{m}$

in the long acyl chain ${ }^{30,31}$. In addition, protein presents a prominent Raman band at $2930 \mathrm{~cm}^{-1}$ due to strong vibration of symmetric $\mathrm{CH}_{3}$. To verify the SRS spectra, Fig. $5 \mathrm{~d}$ illustrates the corresponding spontaneous Raman spectra which are consistent with the major features found in the SRS spectra.

We further examined unprocessed mouse brain tissue by a hyperspectral visible SRS microscope. A stack of 45 SRS images $(800 \times 800$ pixels per image $)$ was obtained at wavenumbers ranging from 2795 to $3035 \mathrm{~cm}^{-1}$. Thus, we were able to record the Raman spectra and analyze the composition of the tissue at each pixel of the highresolution image (SRS image at $2920 \mathrm{~cm}^{-1}$ is presented in Fig. 5c). Figure 5e shows the SRS spectra collected at pinpointed locations in Fig. 5c, where a myelin sheath of axons (circled in purple), neuronal nucleus (green) and nucleolus (red) can be seen. As previously discussed, the lipid droplets largely distributed in the cultured neurons were seldom found in brain tissue. Meanwhile, the Raman spectra for the nucleus and nucleolus presented a strong peak at $2930 \mathrm{~cm}^{-1}$, suggesting that their dominant contents are proteins. As expected, we specifically observed the signature Raman band of sphingomyelin at $2883 \mathrm{~cm}^{-1}$ at the locations of all the axons (Fig. 5e, blue), which implies that the insulating layers of myelinated axons are densely wrapped up with saturated fat, sphingomyelin. In further investigations, we also found that the myelin sheath contained unsaturated fat along with saturated fat at some locations, as shown in Fig. 5f, where the spectra show an emergent Raman band of $3005 \mathrm{~cm}^{-1}$. This band is possibly due to the existence of some amount of phosphatidylethanolamine, phosphatidylserine, or other unsaturated fats in a typical myelin sheath ${ }^{32}$. However, further verification regarding the chemical contents of the myelin sheath is required.

\section{Discussion}

In summary, we developed label-free visible SRS microscopy with a spatial resolution near $130 \mathrm{~nm}$ and demonstrated high-resolution imaging of lowconcentration endogenous biomolecules in cells and tissues with great sensitivity. Moreover, optical fiber-based spectral focusing technology enabled high-resolution biochemical analysis for intact tissues. For further research, most potential applications of visible SRS microscopy can be extended to imaging widely available visible dyes, other than NIR dyes, for supermultiplexing microscopy. 
a

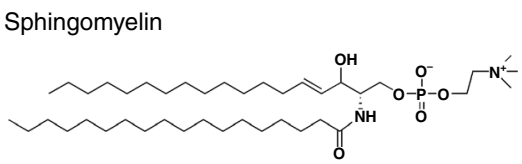

C

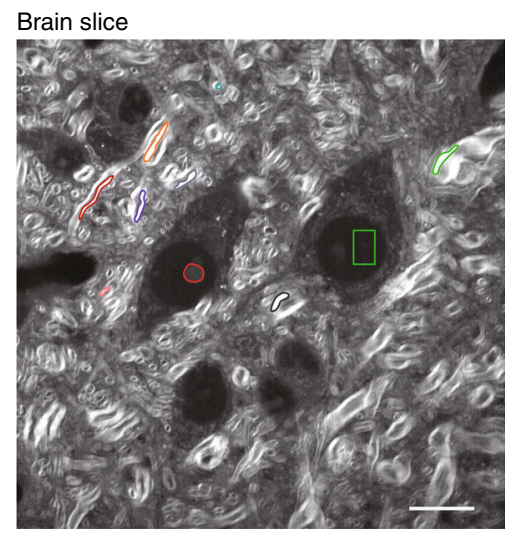

e

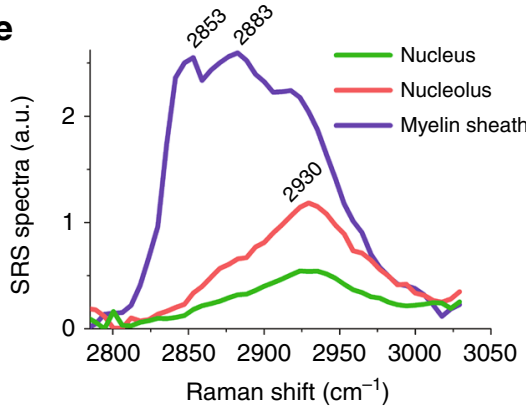

b

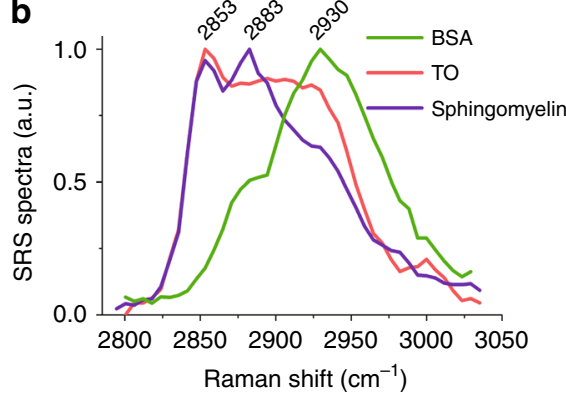

d

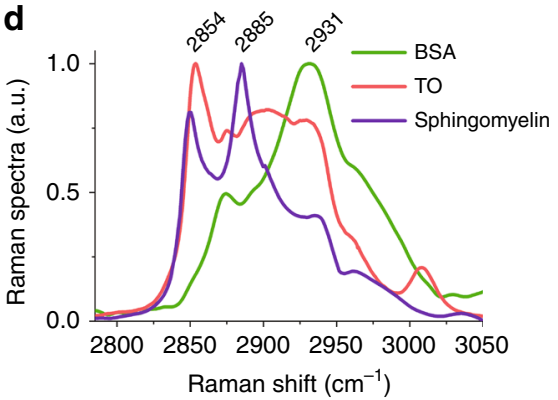

f

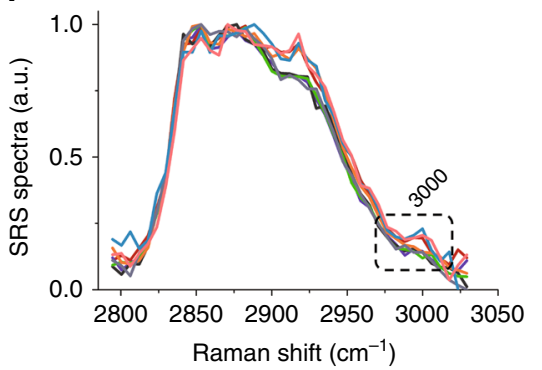

Fig. 5 Hyperspectral visible SRS imaging of mouse brain tissue. a Chemical structure of sphingomyelin. b SRS spectra for BSA, TO, and sphingomyelin in the Raman region of $2795-3035 \mathrm{~cm}^{-1}$. c Hyperspectral visible SRS image stack of a brain slice. Single SRS image at a Raman shift of $2,920 \mathrm{~cm}^{-1}$ is shown. $\mathbf{d}$ Spontaneous Raman spectra corresponding to (b). e SRS spectra for a myelin sheath, nucleus, and nucleolus acquired at the locations indicated in (c). f SRS spectra for myelin sheaths at different locations circled in (c). Scale bar, $10 \mu \mathrm{m}$

\section{Materials and methods}

Visible stimulated Raman scattering microscopy

We employed a dual-output femtosecond laser system (InSight DeepSee, Spectra-Physics, Newport) to provide two phase-locked femtosecond lasers with a repetition rate of $80 \mathrm{MHz}$. The $220 \mathrm{fs}$ output laser at a wavelength of 1040 nm was modulated by an acousto-optical modulator (AOM, $1205 \mathrm{C}-1$, Isomet) at $2.185 \mathrm{MHz}$ with $100 \%$ modulation (the first diffraction order of AOM was adopted). To generate short laser wavelengths for visible SRS imaging, we implemented a BBO crystal $(4 \times 4 \times 0.8 \mathrm{~mm}$, SHG@1040 nm, Union Optic) to double the frequency of the $1040 \mathrm{~nm}$ laser to $520 \mathrm{~nm}$, which served as a Stokes beam. Similarly, the second $100 \mathrm{fs}$ laser at $900 \mathrm{~nm}$ was frequency doubled by the second BBO crystal $(4 \times 4 \times 0.6 \mathrm{~mm}$, SHG@750-1100 nm, Castech) to a $450 \mathrm{~nm}$ laser, which served as a pump beam. A time delay device based on a motorized stage (TLS28EKT04U/TDC15, Zaber) was applied to obtain SRS spectra in spectral focusing. After spatially overlapping the pump and Stokes lasers through a dichroic mirror (ZT491dcsp, Chroma Technology), both lasers were coupled into a 0.3-m-long optical fiber (LPC-04-450-3/125-P1.6-10AC-40-3AF-3-0.3, OZ Optics) to chirp the pulse width for spectral focusing. The two color collinear Gaussian beams were guided into a custom-built inverted microscope equipped with a two-axis galvanometer (GVS002, Thorlabs). For high-resolution imaging, we used three high N.A. objectives (N.A. 1.2, UPLSAPO 60XW; N. A. 1.1, LUMFLN 60XW; N.A. 1.49, UAPON 100XOTIRF, Olympus) in various experiments. An objective scanner (P725.4CD, Physik Instrumente) was installed for 3D cell imaging. After interaction with samples, the transmission lasers were collected by a high N.A. oil condenser (N.A. 1.4, U-AAC, Olympus) and detected by a Si PIN photodiode (S3994-01, Hamamatsu), which was equipped with a 2.1 $\mathrm{MHz}$ resonant amplifier. To block the modulated $520 \mathrm{~nm}$ 
laser, two high-quality bandpass filters (ET450/40M/2P, Chroma Technology) were installed in front of the detector. To demodulate the visible SRS signal, we implemented a high-speed lock-in amplifier (HF2 LI, Zurich Instruments) for sensitive heterodyne detection. For fluorescence imaging, a $450 \mathrm{~nm}$ pump laser was applied as excitation, with two bandpass filters (ET525/50m-2p, ET525/70m-2p, Chroma Technology) placed before a photomultiplier tube (H7422-40, Hamamatsu) used to block all scattering photons except for fluorescence. All imaging signals were acquired by a $1.25 \mathrm{MHz}$ acquisition card (PCI $6251 \mathrm{NI}$, National Instrument).

\section{Laser settings and data acquisition}

The laser powers were measured before the objective for all experiments. For PS bead imaging in Supplementary Fig. 1a, c, the pump and Stokes lasers were set at 792 $\mathrm{nm}(30 \mathrm{~mW})$ and $1040 \mathrm{~nm}(45-55 \mathrm{~mW})$, respectively. As shown in Supplementary Fig. 1a, the dwell time for NIR SRS imaging was $10 \mu \mathrm{s} /$ pixel, with a field of view of $80 \times$ $80 \mu \mathrm{m}^{2}$ with $2000 \times 2000$ pixels. As shown in Supplementary Fig. 1c, the field of view was $40 \times 40 \mu \mathrm{m}^{2}$ with $1600 \times 1600$ pixels, and the dwell time for imaging was 50 $\mu \mathrm{s} /$ pixel. For PS bead imaging in the visible SRS system (Fig. 1c, Supplementary Fig. 1e), we applied an oil immersion objective $(100 \times$, N.A. 1.49). The pump and Stokes lasers were set at $449.5 \mathrm{~nm}(1.7 \mathrm{~mW})$ and $520 \mathrm{~nm}$ $(1.6 \mathrm{~mW})$, respectively. The field of view was $20 \times 20 \mu \mathrm{m}^{2}$ with $1600 \times 1600$ pixels, and the dwell time was $10 \mu \mathrm{s} /$ pixel. For visible SRS imaging of biological samples, the laser powers were 3-5 $\mathrm{mW}$ for both the pump and Stokes beams. The dwell times and pixel settings were $20 \mu \mathrm{s} /$ pixel, $2000 \times 2000$ pixels for U2OS cells (Fig. 2d, e, Supplementary Fig. 5); $40 \mu \mathrm{s} /$ pixel, $1600 \times 1600$ pixels for neurons (Fig. 2a, b, Supplementary Fig. 4c, d); $30 \mu s /$ pixel, $1600 \times 1600$ pixels for HeLa cells (Fig. 3, Supplementary Fig. 6, Supplementary Video); and $35 \mu$ s/pixel, $2000 \times$ 2000 pixels for brain tissue (Figs. 4, 5, Supplementary Figs. 7, 9 and 8d-f). For the fluorescence imaging in Supplementary Fig. 4a, b, the pixel setting is $800 \times 800$ pixels with a dwell time of $10 \mu \mathrm{s} /$ pixel. For the cellular viability test in Supplementary Fig. 2, we used dwell times of $10 \mu \mathrm{s} /$ pixel, with a field of view of $100 \times 100 \mu^{2}$ with $800 \times 800$ pixels per frame. For the NIR SRS imaging in Supplementary Fig. 8a-c, the power of both pump and Stokes beams was $40 \mathrm{~mW}$, and the pixel setting was $400 \times$ 400 pixels with dwell time of $10 \mu \mathrm{s} /$ pixel. For hyperspectral SRS imaging of brain tissue (Fig. 5), we applied a water immersion objective with N.A. 1.2, with pump and Stokes beam laser powers of 4.2 and $2.6 \mathrm{~mW}$, respectively.

\section{Sensitivity analysis for visible SRS imaging}

When the energy difference between the pump and Stokes photons matches the vibrational energy of the target molecules, the coherently induced vibrational transition absorbs one photon (stimulated Raman loss $(\mathrm{SRL})$ ) in the pump beam and gains one photon in the Stokes beam (stimulated Raman gain (SRG)). The signal for SRL can be expressed by

$$
\Delta I_{p} \propto-N \times \sigma_{\text {Raman }} \times I_{p} \times I_{S}
$$

Here, $\Delta I_{\mathrm{p}}$ denotes the relative loss in the pump laser, $N$ is the number of vibrational bonds in the imaging volume, $\sigma_{\text {Raman }}$ is the Raman scattering cross-section, and $I_{\mathrm{p}}, I_{\mathrm{S}}$ are the laser intensities of the pump and Stokes beam, respectively ${ }^{15}$.

Importantly, $\sigma_{\text {Raman }}$ can be significantly increased by near resonant condition. $\sigma_{\text {Raman }}$ can be described as ${ }^{33-35}$

$$
\sigma_{\text {Raman }}=K \omega_{\text {pump }} \omega_{\text {Stokes }}^{3}\left[\frac{\omega_{\text {pump }}^{2}+\omega_{e}^{2}}{\left(\omega_{e}^{2}-\omega_{\text {pump }}^{2}\right)^{2}}\right]^{2}
$$

Here, $\omega_{\text {pump }}$ and $\omega_{\text {Stokes }}$ are the pump and Stokes laser frequencies, respectively; $\omega_{\mathrm{e}}$ is the frequency of the laser that can drive an electronic transition, and $K$ is a constant related to the target biomolecules. Obviously, for $\omega_{\text {pump }}$ " $\omega_{\mathrm{e}}, \sigma_{\text {Raman }}$ will increase by 16 times after frequency doubling. In typical UV absorption spectra for biomolecules, lipids or fatty acids are responsible for absorption at $\sim 150$ $\mathrm{nm}$ due to the $\sigma \rightarrow \sigma^{*}$ transition of the carbon-hydrogen vibrations. DNA and proteins show different but strong absorption bands at $\sim 260$ and $280 \mathrm{~nm}^{36}$, which are due to the $n \rightarrow \pi$ " transition of the aromatic groups.

According to formula (2), $\sigma_{\text {Raman }}$ actually increases by 23 times, as the energy of the electronic transition corresponds to $150 \mathrm{~nm}$. Meanwhile, the intensity of the pump and Stokes lasers $\left(I_{\mathrm{p}}, I_{\mathrm{S}}\right)$ can increase 4 times each because of the smaller focal spot. Moreover, the molecule number $N$ will decrease 8 times due to reduced focal volume. Thus, the SRS intensity $\left(\Delta I_{\mathrm{p}}\right)$ of visible SRS is approximately 46 times more than that of an NIR laserbased SRS. Considering that the shot noise proportionally increases with photon energy, the factor of the sensitivity enhancement should be 23 .

\section{Sample preparation for visible SRS and fluorescence imaging \\ Pure samples}

Droplets of DMSO (30072418, Sinopharm Chemical Reagent Co., Ltd), MeOH (10014118, Sinopharm Chemical Reagent Co., Ltd), FA (dissolved in double steamed water, F7876, Sigma-Aldrich), TO (T7140, SigmaAldrich), powder of BSA (4240GR005, BioFroxx), and sphingomyelin (S0756, Sigma-Aldrich) were sealed between two cover glasses (48393-172, VWR) for different experiments. 


\section{Visible SRS imaging of PS beads}

PS beads $(0.06 \mu \mathrm{m}$ mean particle size, Spherotech, Inc.) were resuspended by vortexing with deionized water diluted to an appropriate concentration. Then, the solution of PS beads was sonicated for $20 \mathrm{~min}$ at room temperature to obtain a homogeneous solution, followed by a 1:1000 dilution with deionized water ${ }^{37}$. Before visible SRS imaging, the beads were sealed between two glasses. We dried the beads on one cover glass and immersed them in pure oil (glyceryl trioleate, TO) to avoid Brownian motion.

\section{Cell lines}

HeLa cells (Fig. 3, Supplementary Figs. 2, 6, Supplementary Video) were cultured in Dulbecco's modified Eagle's medium (DMEM, Thermo Fisher Scientific, Inc.); the human osteosarcoma cell line (U2OS, Fig. 2d, e, Supplementary Fig. 5) cells were cultured in McCoy's 5A medium (Thermo Fisher Scientific, Inc.). All media were supplemented with $10 \%$ fetal bovine serum (Thermo Fisher Scientific, Inc.) and $1 \%$ penicillin-streptomycin (BasalMedia) and maintained in a humidified atmosphere with $5 \% \mathrm{CO}_{2}$ at $37^{\circ} \mathrm{C}^{38}$.

\section{Visible SRS imaging of fixed HeLa and U2OS cells}

$\mathrm{HeLa}$ and U2OS cells were seeded in Petri dishes (glass bottom $\Phi 20 \mathrm{~mm}$, Cellvis) at a density of $1 \times 10^{5}$ per well with $1 \mathrm{ml}$ DMEM/McCoy's $5 \mathrm{~A}$ medium for $24 \mathrm{~h}$ and then fixed for $10 \mathrm{~min}$ at room temperature with $4 \%$ paraformaldehyde (PFA) ${ }^{39}$. After fixation, cells were washed three times with phosphate-buffered saline (PBS, pH of 7.4, Gibco) before imaging.

\section{Mice and tissues}

We have complied with all relevant ethical regulations, and all animal experiments were approved by the Institutional Animal Ethics Committee of Huazhong University of Science and Technology. The brain tissue from a 4-week-old C57BL/6J female mouse (Figs. 4, 5, Supplementary Figs. 7, 9 and 8d-f) was sliced and washed once by PBS before sealing between two coverslips. The thickness of the tissue slice is $50 \mu \mathrm{m}$ in Fig. 4 and $70 \mu \mathrm{m}$ in Fig. 5.

\section{Immunofluorescence staining of fixed neurons}

Neurons (Supplementary Fig. 4a, b) cultured on glass coverslips in 24-well plates were fixed with 4\% PFA for 10 $\mathrm{min}$, and then washed with PBS three times. After a permeabilization process with $0.2 \%$ Triton X-100 for $10 \mathrm{~min}$, all neurons were treated with a blocking solution (3\% BSA in PBS) for $30 \mathrm{~min}$ and rinsed twice with $\mathrm{PBS}^{40}$. The primary antibody, anti-MAP2 antibody in rabbit (ab32454, Abcam), was diluted by 1:1000 in a 3\% BSA solution and added into plates overnight at $4{ }^{\circ} \mathrm{C}$. Then, the neurons were rinsed three times with PBS. A goat-anti-rabbit Alexa488 (ab150077, Abcam) secondary antibody was applied followed by a 1:500 dilution in 3\% BSA solution, and cells were incubated for $1 \mathrm{~h}$ at room temperature in the dark ${ }^{33}$. These glass coverslips were moved to Petri dishes (glass bottom $\Phi$ $20 \mathrm{~mm}$, Cellvis) filled with PBS. Neurons were washed three times with PBS before imaging.

\section{Data availability}

The data that support the findings of this study are available from the corresponding authors upon request.

\section{Acknowledgements}

We thank Jihong Gong and Cong Ma for providing neurons, Bo Xin and Zhenli Huang for providing HeLa cells and the oil objective, Wenting Zhang and Yuhui Zhang for providing U2OS cells, Ruixi Chen and Saoqun Zeng for providing brain slices, and Saoqun Zeng and Liang Luo for helpful discussions. We thank the Analytical \& Testing Center of HUST for the Raman spectrum measurements. P.W. acknowledges support from the National Key Research and Development Program of China (2016YFA0201403), National Natural Science Foundation of China (61675075), Science Fund for Creative Research Group of China (61421064), Chinese Recruitment Program of Global Experts, and Director Fund of the Wuhan National Laboratory for Optoelectronics. G.Z. is funded by the Natural Science Foundation of Hubei Province (grant 2017CFB591).

\section{Author details \\ 'Britton Chance Center for Biomedical Photonics, Wuhan National Laboratory for Optoelectronics-Huazhong University of Science and Technology, Wuhan, Hubei 430074, China. ${ }^{2}$ MoE Key Laboratory for Biomedical Photonics, Collaborative Innovation Center for Biomedical Engineering, School of Engineering Sciences, Huazhong University of Science and Technology, Wuhan, Hubei 430074, China. ${ }^{3}$ MoE Key Laboratory of Quark and Lepton Physics and College of Physics Science and Technology, Central China Normal University, Wuhan 430079, China}

\section{Authors' contributions}

Y.B. and C.Y. carried out the construction of the visible SRS imaging system and performed experiments. Y.B., C.Y., and P.W. analyzed the data. Y.B., C.Y., and Y.C. prepared the biological samples. Y.B., C.Y., and P.W. wrote the manuscript with input from all authors. P.W., Y.B., and C.Y. conceived the concept. P.W. and G.Z. supervised the project.

Conflict of interest

The authors declare that they have no conflict of interest.

Supplementary information is available for this paper at https://doi.org/ 10.1038/s41377-018-0082-1.

Received: 29 June 2018 Revised: 21 September 2018 Accepted: 2 October 2018 Accepted article preview online: 8 October 2018

Published online: 24 October 2018

\section{References}

1. Yang, Y. L. et al. Differential diagnosis of breast cancer using quantitative, labelfree and molecular vibrational imaging. Biomed. Opt. Express 2, 2160-2174 (2011).

2. Mittal, R. et al. Evaluation of stimulated Raman scattering microscopy for identifying squamous cell carcinoma in human skin. Lasers Surg. Med. 45, 496-502 (2013).

3. Uckermann, O. et al. Label-free delineation of brain tumors by coherent antistokes Raman scattering microscopy in an orthotopic mouse model and human glioblastoma. PLOS ONE 9, e107115 (2014).

4. Hering, K. et al. SERS: a versatile tool in chemical and biochemical diagnostics. Anal. Bioanal. Chem. 390, 113-124 (2008). 
5. Kim, H., Bryant, G. W. \& Stranick, S. J. Superresolution four-wave mixing microscopy. Opt. Express 20, 6042-6051 (2012).

6. Wang, $Y$. et al. Wide-field, surface-sensitive four-wave mixing microscopy of nanostructures. Appl. Opt. 51, 3305-3312 (2012).

7. Liu, X. J., Wang, Y. \& Potma, E. O. Surface-mediated four-wave mixing of nanostructures with counterpropagating surface plasmon polaritons. Opt. Lett. 36, 2348-2350 (2011).

8. Zumbusch, A., Holtom, G. R. \& Xie, X. S. Three-dimensional vibrational imaging by coherent anti-Stokes Raman scattering. Phys. Rev. Lett. 82, 4142-4145 (1999).

9. Evans, C. L. \& Xie, X. S. Coherent anti-Stokes Raman scattering microscopy: chemical imaging for biology and medicine. Annu Rev. Anal. Chem. 1, 883-909 (2008).

10. Camp, C. H. Jr et al. High-speed coherent Raman fingerprint imaging of biological tissues. Nat. Photonics 8, 627-634 (2014).

11. Yampolsky, S. et al. Seeing a single molecule vibrate through time-resolved coherent anti-Stokes Raman scattering. Nat. Photonics 8, 650-656 (2014)

12. Liu, W. \& Niu, H. B. Diffraction barrier breakthrough in coherent anti-Stokes Raman scattering microscopy by additional probe-beam-induced phonon depletion. Phys. Rev. A 83, 023830 (2011).

13. Kawata S., Ichimura T., Hayazawa N., Hashimoto M., Inouye Y. Tip-enhanced near-field CARS microscopy for molecular nano-imaging. Proceedings of SPIE 5700, Multiphoton Microscopy in the Biomedical Sciences V. 5700: 52-59 (SPIE, San Jose, 2005).

14. Cheng, J. X. \& Xie, X. S. Vibrational spectroscopic imaging of living systems: an emerging platform for biology and medicine. Science 350, aaa8870 (2015).

15. Freudiger, C. W. et al. Label-free biomedical imaging with high sensitivity by stimulated Raman scattering microscopy. Science 322, 1857-1861 (2008).

16. Hell, S. W. Toward fluorescence nanoscopy. Nat. Biotechnol. 21, 1347-1355 (2003).

17. Duncan, M. D., Reintjes, J. \& Manuccia, T. J. Scanning coherent anti-Stokes Raman microscope. Opt. Lett. 7, 350-352 (1982).

18. Houston, W. V. A compound interferometer for fine structure work. Phys. Rev. 29, 478-484 (1927).

19. Hell, S. W. Far-field optical nanoscopy. Science 316, 1153-1158 (2007).

20. Allen, K. W. et al. Overcoming the diffraction limit of imaging nanoplasmonic arrays by microspheres and microfibers. Opt. Express 23, 24484-24496 (2015).

21. Fu, D. et al. Quantitative chemical imaging with multiplex stimulated Raman scattering microscopy. J. Am. Chem. Soc. 134, 3623-3626 (2012).

22. Ozeki, Y. et al. High-speed molecular spectral imaging of tissue with stimulated Raman scattering. Nat. Photonics 6, 845-851 (2012).

23. Fu, D., Holtom, G., Freudiger, C., Zhang, X. \& Xie, X. S. Hyperspectral imaging with stimulated Raman scattering by chirped femtosecond lasers. J. Phys. Chem. B 117, 4634-4640 (2013).
24. Paxinos G., Franklin K. B. J. The Mouse Brain in Stereotaxic Coordinates. 2nd edn. (Academic Press, San Diego, 2001).

25. Ji, M. B. et al. Rapid, label-free detection of brain tumors with stimulated Raman scattering microscopy. Sci. Transl. Med. 5, 201 ra119 (2013).

26. Fu, D. et al. In vivo metabolic fingerprinting of neutral lipids with hyperspectral stimulated Raman scattering microscopy. J. Am. Chem. Soc. 136, 8820-8828 (2014).

27. Heinrich, $C$. et al. Selective imaging of saturated and unsaturated lipids by wide-field CARS-microscopy. Opt. Express 16, 2699-2708 (2008).

28. Di Napoli, C. et al. Quantitative spatiotemporal chemical profiling of individual lipid droplets by hyperspectral CARS microscopy in living human adiposederived stem cells. Anal. Chem. 88, 3677-3685 (2016).

29. Slipchenko, M. N., Le, T. T., Chen, H. T. \& Cheng, J. X. High-speed vibrational imaging and spectral analysis of lipid bodies by compound Raman microscopy. J. Phys. Chem. B 113, 7681-7686 (2009).

30. Rinia, H. A., Burger, K. N. J., Bonn, M. \& Müller, M. Quantitative label-free imaging of lipid composition and packing of individual cellular lipid droplets using multiplex CARS microscopy. Biophys. J. 95, 4908-4914 (2008).

31. Wu, H. W. et al. In vivo lipidomics using single-cell Raman spectroscopy. Proc. Natl Acad. Sci. USA 108, 3809-3814 (2011).

32. Krafft, C., Neudert, L., Simat, T. \& Salzer, R. Near infrared Raman spectra of human brain lipids. Spectrochim. Acta A Mol. Biomol. Spectrosc. 61, 1529-1535 (2005).

33. Wei, L. et al. Super-multiplex vibrational imaging. Nature 544, 465-470 (2017).

34. Albrecht, A. C. \& Hutley, M. C. On the dependence of vibrational Raman intensity on the wavelength of incident light. J. Chem. Phys. 55, 4438-4443 (1971).

35. Asher, S. A. UV resonance Raman studies of molecular structure and dynamics: applications in physical and biophysical chemistry. Annu. Rev. Phys. Chem. 39, 537-588 (1988).

36. Held P. Quantitation of Peptides and Amino Acids with a Synergy ${ }^{\mathrm{TM}}$ HT Using UV Fluorescence (B.-T. Instruments, Winooski, 2003).

37. Jin, C., Nadakuditi, R. R., Michielssen, E. \& Rand, S. C. Iterative, backscatteranalysis algorithms for increasing transmission and focusing light through highly scattering random media. J. Opt. Soc. Am. A 30, 1592-1602 (2013).

38. Han, Y. B., Li, M. H., Qiu, F. W., Zhang, M. \& Zhang, Y. H. Cell-permeable organic fluorescent probes for live-cell long-term super-resolution imaging reveal lysosome-mitochondrion interactions. Nat. Commun. 8, 1307 (2017).

39. Pan, D. et al. A general strategy for developing cell-permeable photo-modulatable organic fluorescent probes for live-cell super-resolution imaging. Nat. Commun. 5, 5573 (2014).

40. Matsuzaki, F., Shirane, M., Matsumoto, M. \& Nakayama, K. I. Protrudin serves as an adaptor molecule that connects KIF5 and its cargoes in vesicular transport during process formation. Mol. Biol. Cell 22, 4602-4620 (2011). 